\title{
正规化双全纯映射精细的 展开式系数估计*
}

\author{
刘小松 刘太顺 ${ }^{* *}$
}

(中国科学技术大学数学系, 合肥 230026)

摘要 在 $\mathbb{C}^{n}$ 中的单位多圆柱上或复 Banach 空间的单位球上讨论正规化双全 纯映射子族中映射 $f(x=0$ 是 $f(x)-x$ 的 $k+1$ 阶零点) 的齐次展开式的精细估 计. 并且, 在复 Banach 空间的单位球上也给出了一类从属映射的齐次展开式的 估计.

关键词 de Branges 定理 齐次展开式的精细估计 $\alpha$ 次殆星形映射 $\alpha$ 次星形映射 从属映射

在单复变数情形, 下面的 de Branges 定理是众所周知的:

定理 $\mathbf{A}^{[1]}$ 若 $f(z)=z+\sum_{n=2}^{\infty} a_{n} z^{n}$ 是单位圆盘 $D$ 上的双全纯函数, 则

$$
\left|a_{n}\right| \leqslant n, \quad n=2,3, \cdots .
$$

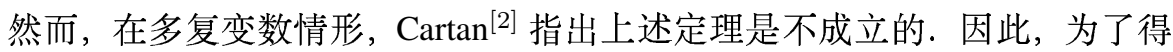
到一些正面的结果必须对映射加上一些附加的条件，如凸性、星形性等.

遗憾的是, 迄今为止多复变数中关于正规化双全纯映射子族的齐次展开式 的估计只有一些零星的结果 (参见文献 $[1,3,4]$ ). 特别地, 在 $\mathbb{C}^{n}$ 中的单位多圆柱 $D^{n}$ 上, 关于正规化双全纯星形映射的齐次展开式的估计, 龚升 ${ }^{[1]}$ 提出了下面的

猜想 若 $f: D^{n} \rightarrow \mathbb{C}^{n}$ 是正规化双全纯星形映射, 其中 $D^{n}$ 是 $\mathbb{C}^{n}$ 中的单位 多圆柱, 则

$$
\frac{\left\|D^{m} f(0)\left(z^{m}\right)\right\|}{m !} \leqslant m\|z\|^{m}, \quad z \in D^{n}, \quad m=2,3, \cdots .
$$

目前, 仅证明了 $m=2$ 时, 猜想是成立的 ${ }^{[1]}$. 而在 $\mathbb{C}^{n}$ 中的 Euclid 单位球 $B^{n}$ 上, Roper 和 Suffridge ${ }^{[5]}$ 举出一个反例表明上述猜想在 $m=2$ 时就不成立. 这就 
是人们主要在 $\mathbb{C}^{n}$ 中的单位多圆柱 $D^{n}$ 上研究正规化双全纯映射子族齐次展开式 的估计的原因.

设 $X$ 是具有范数 $\|\cdot\|$ 的复 Banach 空间, $X^{*}$ 是 $X$ 的对偶空间, $B$ 是 $X$ 中 的开单位球, $D$ 为 $\mathbb{C}$ 中的 Euclid 开单位圆盘, $D^{n}$ 表示 $\mathbb{C}^{n}$ 中的开单位多圆柱, $B^{n}$ 是 $\mathbb{C}^{n}$ 中的 Euclid 开单位球; $\partial B$ 是 $B$ 的边界, $\bar{B}$ 为 $B$ 的闭包, $\partial_{0} D^{n}$ 是 $D^{n}$ 的特征边界 (即全纯函数的模能取到最大值的边界). $\mathbb{N}$ 表示正整数集, $\mathbb{R}$ 表示实 数集. 对于任意 $x \in X \backslash\{0\}$, 定义 $T(x)=\left\{T_{x} \in X^{*}:\left\|T_{x}\right\|=1, T_{x}(x)=\|x\|\right\}$. 根据 Hahn-Banach 定理, $T(x)$ 非空. 任意 $\alpha(\neq 0) \in \mathbb{C}$, 因 $\frac{|\alpha|}{\alpha} T_{x} \in T(\alpha x)$ 对应 $T_{x} \in T(x)$, 故总用 $T_{\alpha x}$ 表示 $\frac{|\alpha|}{\alpha} T_{x}$. 将 $B$ 映到 $X$ 内的全纯映射的全体记为 $H(B)$, 而将 $B$ 映 到 $B$ 内的全纯映射的全体记为 $H(B, B)$.

熟知, 若 $f \in H(B)$, 则

$$
f(y)=\sum_{n=0}^{\infty} \frac{1}{n !} D^{n} f(x)\left((y-x)^{n}\right)
$$

在 $x \in B$ 的邻域中的点 $y$ 上成立, 其中 $D^{n} f(x)$ 是 $f$ 在点 $x$ 的第 $n$ 次 Fréchet 导 数, 当 $n \geqslant 1$ 时,

$$
D^{n} f(x)\left((y-x)^{n}\right)=D^{n} f(x)(\underbrace{y-x, \cdots, y-x}_{n}) .
$$

而且, $D^{n} f(x)$ 是将 $\prod_{j=1}^{n} X$ 映到 $X$ 内的有界对称 $n$ - 线性映射.

若全纯映射 $f: B \rightarrow X$ 的逆映射存在, 且在开集 $f(B)$ 上全纯, 则称 $f$ 在 $B$ 上是双全纯的. 如果全纯映射 $f$ 的 Fréchet 导数 $D f(x)$ 在每点 $x \in B$ 处都存在 有界的逆, 则称 $f$ 在 $B$ 上是局部双全纯的. $B$ 上的全纯映射 $f: B \rightarrow X$ 若满足 $f(0)=0, D f(0)=I$, 则称 $f$ 是正规化的, 其中 $I$ 是将 $X$ 映到 $X$ 内的单位算子.

定义 1 设 $0 \leqslant \alpha<1$, 且 $f: B \rightarrow X$ 是正规化局部双全纯映射. 若

$$
\operatorname{Re}\left\{T_{x}\left[(D f(x))^{-1} f(x)\right]\right\} \geqslant \alpha\|x\|, \quad \forall x \in B,
$$

则称 $f$ 是 $B$ 上的 $\alpha$ 次殆星形映射.

若 $X=\mathbb{C}^{n}, B=D^{n}$, 则上述条件显然等价于

$$
\operatorname{Re} \frac{g_{j}(z)}{z_{j}} \geqslant \alpha, \quad \forall z \in D^{n},
$$

其中 $g(z)=\left(g_{1}(z), \cdots, g_{n}(z)\right)^{\prime}=(D f(z))^{-1} f(z)$ 是 $\mathbb{C}^{n}$ 中的列向量, $\left|z_{j}\right|=\|z\|=$ $\max _{1 \leqslant k \leqslant n}\left\{\left|z_{k}\right|\right\}$.

若 $\alpha=0$, 则定义 1 给出 $B$ 上正规化局部双全纯映射成为星形映射的判别准 则 (见文献 [6]).

定义 $2^{[7]}$ 设 $0<\alpha<1$, 且 $f: B \rightarrow X$ 是正规化局部双全纯映射. 若

$$
\left|\frac{1}{\|x\|} T_{x}\left[(D f(x))^{-1} f(x)\right]-\frac{1}{2 \alpha}\right|<\frac{1}{2 \alpha}, \quad \forall x \in B \backslash\{0\},
$$

则称 $f$ 是 $B$ 上的 $\alpha$ 次星形映射. 
若 $X=\mathbb{C}^{n}, B=D^{n}$, 则上述条件显然等价于

$$
\left|\frac{g_{j}(z)}{z_{j}}-\frac{1}{2 \alpha}\right|<\frac{1}{2 \alpha}, \quad \forall z \in D^{n} \backslash\{0\},
$$

其中 $g(z)=\left(g_{1}(z), \cdots, g_{n}(z)\right)^{\prime}=(D f(z))^{-1} f(z)$ 是 $\mathbb{C}^{n}$ 中的列向量, $\left|z_{j}\right|=\|z\|=$ $\max _{1 \leqslant k \leqslant n}\left\{\left|z_{k}\right|\right\}$.

定义 $3^{[8]}$ 设 $f: B \rightarrow X$ 是一个双全纯映射. 若存在 $\varepsilon \in[0,1]$, 使得 $f(B)$ 相 对于 $\varepsilon f(B)$ 的每一点而言都是星形的, 则称 $f$ 是 $B$ 上的 $\varepsilon$ 星形映射, $B$ 上 $\varepsilon$ 星形 映射的全体组成 $\varepsilon$ 星形映射族.

当 $\varepsilon=0$ 时, 这是星形映射族; 而当 $\varepsilon=1$ 时, 这就是凸映射族.

定义 4 $^{[9]}$ 设 $f: B \rightarrow X$ 是正规化局部双全纯映射. 若

$$
\operatorname{Re}\left\{T_{x}\left[(D f(x))^{-1}(f(x)-f(\xi x))\right]\right\} \geqslant 0, \quad \forall x \in B, \quad \xi \in \bar{D},
$$

则称 $f$ 是 $B$ 上的准凸映射.

$B$ 上正规化双全纯凸映射 (星形映射) 的全体记为 $K(B)\left(S^{*}(B)\right), B$ 上准凸 映射的全体记为 $Q(B), B$ 上 $\alpha$ 次星形映射的全体记为 $S_{\alpha}^{*}(B)$.

定义 $5^{[10]}$ 设 $f \in H(B)$. 若 $f(0)=0, \cdots, D^{k-1} f(0)=0$, 但 $D^{k} f(0) \neq 0$, 其 中 $k \in \mathbb{N}$, 则称 $x=0$ 是 $f(x)$ 的 $k$ 阶零点. 当 $X=\mathbb{C}$ 时, 这与通常的定义是一致 的.

定义 6 设 $f \in H(B), g \in H(B)$. 若存在映射 $\varphi \in H(B, B), \varphi(0)=0$, 使得 $g(x)=f(\varphi(x))$, 则称 $g$ 从属于 $f($ 记为 $g \prec f)$.

本文的主要结果是下面的定理和推论:

定理 1 若 $f(z)$ 是 $D^{n}$ 上的 $\alpha(0 \leqslant \alpha<1)$ 次殆星形映射, 且 $z=0$ 是 $f(z)-z$ 的 $k+1(k \in \mathbb{N})$ 阶零点, 则

$$
\frac{\left\|D^{m} f(0)\left(z^{m}\right)\right\|}{m !} \leqslant \frac{2(1-\alpha)}{m-1}\|z\|^{m}, \quad z \in D^{n}, \quad m=k+1, \cdots, 2 k .
$$

当 $m=k+1$ 时, 上述估计式是精确的. 特别地, 若 $f(z) \in S^{*}\left(D^{n}\right)$ (即 $\alpha=0$ ), 则

$$
\frac{\left\|D^{m} f(0)\left(z^{m}\right)\right\|}{m !} \leqslant \frac{2}{m-1}\|z\|^{m}, \quad z \in D^{n}, \quad m=k+1, \cdots, 2 k .
$$

当 $m=k+1$ 时, 上述估计式是精确的.

若 $m=2$ (即 $k=1$ ), 则定理 1 是文献 [1] 中相应的结论.

定理 2 若 $f(z) \in S_{\alpha}^{*}\left(D^{n}\right)(0<\alpha<1)$, 且 $z=0$ 是 $f(z)-z$ 的 $k+1(k \in \mathbb{N})$ 阶零点, 则

$$
\frac{\left\|D^{m} f(0)\left(z^{m}\right)\right\|}{m !} \leqslant \frac{2(1-\alpha)}{m-1}\|z\|^{m}, \quad z \in D^{n}, \quad m=k+1, \cdots, 2 k .
$$

当 $m=k+1$ 时, 上述估计式是精确的.

$n=1$ 时, 定理 2 是文献 [11] 中对应结论的部分结果.

定理 3 设 $X$ 是具有内积 $\langle\cdot, \cdot\rangle$ 的复 Hilbert 空间. 若 $f(x) \in K(B), x=0$ 是 $f(x)-x$ 的 $k+1(k \in \mathbb{N})$ 阶零点, 则

$$
\frac{\left\|D^{m} f(0)\left(x^{m}\right)\right\|}{m !} \leqslant \frac{\sin \frac{\pi}{m}}{1+\left(\cos \frac{\pi}{m}\right)^{m}}\|x\|^{m}, \quad x \in B, \quad m=k+1, \cdots, 2 k .
$$


当 $m=2$ (即 $k=1$ ) 时, 上述估计式是精确的.

若 $m=2($ 即 $k=1)$, 则定理 3 是文献 [3] 中对应结论的部分结果.

定理 4 若 $f(x)$ 是 $B$ 上的 $\alpha(0 \leqslant \alpha<1)$ 次殆星形映射, 且 $x=0$ 是 $f(x)-x$ 的 $k+1(k \in \mathbb{N})$ 阶零点, 则

$$
\frac{\left|T_{x}\left(D^{m} f(0)\left(x^{m}\right)\right)\right|}{m !} \leqslant \frac{2(1-\alpha)}{m-1}\|x\|^{m}, \quad x \in B, \quad m=k+1, \cdots, 2 k .
$$

当 $m=k+1$ 时, 上述估计式是精确的. 特别地, 若 $f(x) \in S^{*}(B)$ (即 $\alpha=0$ ), 则

$$
\frac{\left|T_{x}\left(D^{m} f(0)\left(x^{m}\right)\right)\right|}{m !} \leqslant \frac{2}{m-1}\|x\|^{m}, \quad x \in B, \quad m=k+1, \cdots, 2 k .
$$

当 $m=k+1$ 时, 上述估计式是精确的.

定理 5 若 $f(x) \in S_{\alpha}^{*}(B)(0<\alpha<1)$, 且 $x=0$ 是 $f(x)-x$ 的 $k+1(k \in \mathbb{N})$ 阶零点, 则

$$
\frac{\left|T_{x}\left(D^{m} f(0)\left(x^{m}\right)\right)\right|}{m !} \leqslant \frac{2(1-\alpha)}{m-1}\|x\|^{m}, \quad x \in B, \quad m=k+1, \cdots, 2 k .
$$

当 $m=k+1$ 时，上述估计式是精确的.

若 $X=\mathbb{C}^{n}, B=B^{n}, m=2$ (即 $k=1$ ), 则定理 5 是文献 [4] 中的相应结论; 若 $X=\mathbb{C}$, 则定理 5 是文献 [11] 中对应结论的部分结果.

定理 6 若 $f(x)$ 是 $B$ 上正规化双全纯 $\varepsilon(0 \leqslant \varepsilon \leqslant 1)$ 星形映射, 且 $x=0$ 是 $f(x)-x$ 的 $k+1(k \in \mathbb{N})$ 阶零点, 则

$$
\frac{\left|T_{x}\left(D^{m} f(0)\left(x^{m}\right)\right)\right|}{m !} \leqslant \frac{2(1+\varepsilon)}{m-1+m \varepsilon+(-1)^{m} \varepsilon}\|x\|^{m}, \quad x \in B, \quad m=k+1, \cdots, 2 k .
$$

推论 1 若 $f(x) \in Q(B)$, 且 $x=0$ 是 $f(x)-x$ 的 $k+1(k \in \mathbb{N})$ 阶零点, 则

$$
\frac{\left|T_{x}\left(D^{m} f(0)\left(x^{m}\right)\right)\right|}{m !} \leqslant \frac{4}{2 m-1+(-1)^{m}}\|x\|^{m}, \quad x \in B, \quad m=k+1, \cdots, 2 k .
$$

当 $m=2($ 即 $k=1)$ 时, 上述估计式是精确的.

推论 2 若 $f(x) \in S^{*}(B)$, 且 $x=0$ 是 $f(x)-x$ 的 $k+1(k \in \mathbb{N})$ 阶零点, 则

$$
\frac{\left|T_{x}\left(D^{m} f(0)\left(x^{m}\right)\right)\right|}{m !} \leqslant \frac{2}{m-1}\|x\|^{m}, \quad x \in B, \quad m=k+1, \cdots, 2 k .
$$

当 $m=k+1$ 时, 上述估计式是精确的.

若 $X=\mathbb{C}^{n}, B=B^{n}, m=2$ (即 $k=1$ ), 则推论 2 是文献 [4] 中相应的结论.

定理 7 设 $f(x) \in K(B), g(x)=D f(x) x$. 若 $h(x) \prec g(x)$, 则

$$
\frac{\left\|D^{m} h(0)\left(x^{m}\right)\right\|}{m !} \leqslant m\|x\|^{m}, \quad x \in B, \quad m=1,2, \cdots .
$$

当 $X$ 为复 Hilbert 空间时, 上述估计式是精确的.

若 $X=\mathbb{C}$, 则定理 7 是文献 [12] 中相应的结论.

\section{1 一些引理}

为证明本文的定理, 首先给出几条引理.

易证下面的 
引理 1 设 $p(z) \in H(D), p(0)=1$, 且 $\alpha \in[0,1)$. 若 $\operatorname{Re} p(z) \geqslant \alpha, z \in D$, 则

$$
\frac{\left|p^{(n)}(0)\right|}{n !} \leqslant 2(1-\alpha), \quad n=1,2, \cdots .
$$

上述估计式是精确的.

由引理 1 , 可证下面的

引理 2 设 $g \in H\left(D^{n}\right), g(0)=0, D g(0)=I$, 且 $\alpha \in[0,1)$. 若 $\operatorname{Re} \frac{g_{j}(z)}{z_{j}} \geqslant$ $\alpha, z \in D^{n}$, 其中 $\left|z_{j}\right|=\|z\|=\max _{1 \leqslant k \leqslant n}\left\{\left|z_{k}\right|\right\}$, 则

$$
\frac{\left\|D^{m} g(0)\left(z^{m}\right)\right\|}{m !} \leqslant 2(1-\alpha)\|z\|^{m}, \quad z \in D^{n}, \quad m=2,3, \cdots .
$$

上述估计式是精确的.

证 固定 $z \in D^{n} \backslash\{0\}$, 并记 $z_{0}=\frac{z}{\|z\|}$. 定义 $p_{j}(\xi)=\frac{g_{j}\left(\xi z_{0}\right)\|z\|}{\xi z_{j}}, \xi \in D$, 其中 $\left|z_{j}\right|=\|z\|=\max _{1 \leqslant k \leqslant n}\left\{\left|z_{k}\right|\right\}$. 根据条件, 有 $p_{j} \in H(D), p_{j}(0)=\lim _{\xi \rightarrow 0} \frac{g_{j}\left(\xi z_{0}\right)\|z\|}{\xi z_{j}}=$ $\frac{D g_{j}(0) z_{0}\|z\|}{z_{j}}=1$, 且 $\operatorname{Re} p_{j}(\xi)=\operatorname{Re} \frac{g_{j}\left(\xi z_{0}\right)\|z\|}{\xi z_{j}} \geqslant \alpha, \xi \in D$. 注意到

$$
p_{j}(\xi)=1+\frac{D^{2} g_{j}(0)\left(z_{0}^{2}\right)\|z\|}{2 ! z_{j}} \xi+\cdots+\frac{D^{m} g_{j}(0)\left(z_{0}^{m}\right)\|z\|}{m ! z_{j}} \xi^{m-1}+\cdots,
$$

由引理 1 得

$$
\frac{\left|p_{j}^{(m-1)}(0)\right|}{(m-1) !}=\frac{\left|D^{m} g_{j}(0)\left(z_{0}^{m}\right)\right|}{m !} \leqslant 2(1-\alpha), \quad m=2,3, \cdots .
$$

若 $z_{0} \in \partial_{0} D^{n}$, 则

$$
\frac{\left|D^{m} g_{j}(0)\left(z_{0}^{m}\right)\right|}{m !} \leqslant 2(1-\alpha), \quad j=1,2, \cdots, n .
$$

又因 $D^{m} g_{j}(0)\left(z^{m}\right)$ 是 $\overline{D^{n}}$ 上的全纯函数, 根据单位多圆柱上全纯函数的最大模原 理得

$$
\frac{\left|D^{m} g_{j}(0)\left(z_{0}^{m}\right)\right|}{m !} \leqslant 2(1-\alpha), \quad z_{0} \in \partial D^{n}, \quad j=1,2, \cdots, n
$$

即

$$
\frac{\left|D^{m} g_{j}(0)\left(z^{m}\right)\right|}{m !} \leqslant 2(1-\alpha)\|z\|^{m}, \quad z \in D^{n}, \quad j=1,2, \cdots, n,
$$

故

$$
\frac{\left\|D^{m} g(0)\left(z^{m}\right)\right\|}{m !} \leqslant 2(1-\alpha)\|z\|^{m}, \quad z \in D^{n}
$$

证毕.

不难验证 $g(z)=\left(\frac{\left(1-(1-2 \alpha) z_{1}\right) z_{1}}{1+z_{1}}, z_{2}, \cdots, z_{n}\right)^{\prime}$ 满足引理 2 的条件. 取 $z_{1}=$ $r(0 \leqslant r<1), z_{j}=0, j=2,3, \cdots, n$, 则 (1.1) 式中等号成立.

引理 $\mathbf{3}^{[13]}$ 若 $f(z)=a_{0}+\sum_{n=1}^{\infty} a_{n} z^{n} \in H(D)$, 且 $f(D) \subset D$, 则

$$
\left|a_{n}\right| \leqslant 1-\left|a_{0}\right|^{2}, \quad n=1,2, \cdots .
$$

当 $n=1$ 时, 上述估计式是精确的. 
由引理 3, 可证下面的

引理 4 设 $g \in H\left(D^{n}\right), g(0)=0, D g(0)=I$, 且 $\alpha \in(0,1)$. 若 $\left|\frac{g_{j}(z)}{z_{j}}-\frac{1}{2 \alpha}\right|<$ $\frac{1}{2 \alpha}, z \in D^{n} \backslash\{0\}$, 其中 $\left|z_{j}\right|=\|z\|=\max _{1 \leqslant k \leqslant n}\left\{\left|z_{k}\right|\right\}$, 则

$$
\frac{\left\|D^{m} g(0)\left(z^{m}\right)\right\|}{m !} \leqslant 2(1-\alpha)\|z\|^{m}, \quad z \in D^{n}, \quad m=2,3, \cdots .
$$

当 $m=2$ 时, 上述估计式是精确的.

证 固定 $z \in D^{n} \backslash\{0\}$, 并记 $z_{0}=\frac{z}{\|z\|}$. 定义 $p_{j}(\xi)=2 \alpha \frac{g_{j}\left(\xi z_{0}\right)\|z\|}{\xi z_{j}}-1, \xi \in D$, 其中 $\left|z_{j}\right|=\|z\|=\max _{1 \leqslant k \leqslant n}\left\{\left|z_{k}\right|\right\}$. 根据条件, 有 $p_{j} \in H(D), p_{j}(0)=2 \alpha-1$, 且 $p_{j}(D) \subset D$. 注意到

$$
\begin{aligned}
p_{j}(\xi)= & 2 \alpha-1+\frac{2 \alpha D^{2} g_{j}(0)\left(z_{0}^{2}\right)\|z\|}{2 ! z_{j}} \xi \\
& +\cdots+\frac{2 \alpha D^{m} g_{j}(0)\left(z_{0}^{m}\right)\|z\|}{m ! z_{j}} \xi^{m-1}+\cdots, \quad \xi \in D,
\end{aligned}
$$

由引理 3 得

$$
\frac{\left|p_{j}^{(m-1)}(0)\right|}{(m-1) !}=\frac{2 \alpha\left|D^{m} g_{j}(0)\left(z_{0}^{m}\right)\right|}{m !} \leqslant 1-(2 \alpha-1)^{2}, \quad m=2,3, \cdots,
$$

故

$$
\frac{\left|D^{m} g_{j}(0)\left(z_{0}^{m}\right)\right|}{m !} \leqslant 2(1-\alpha), \quad m=2,3, \cdots .
$$

利用与引理 2 相同的证明方法得

$$
\frac{\left\|D^{m} g(0)\left(z^{m}\right)\right\|}{m !} \leqslant 2(1-\alpha)\|z\|^{m}, \quad z \in D^{n} .
$$

证毕.

不难验证 $g(z)=\left(\frac{\left(1-z_{1}\right) z_{1}}{1+(1-2 \alpha) z_{1}}, z_{2}, \cdots, z_{n}\right)^{\prime}$ 满足引理 4 的条件. 取 $z_{1}=r(0 \leqslant$ $r<1), z_{j}=0, j=2,3, \cdots, n$, 则当 $m=2$ 时，(1.2) 式中的等号成立.

由引理 1 , 也可证下面的

引理 5 设 $g \in H(B), g(0)=0, D g(0)=I$, 且 $\alpha \in[0,1)$. 若 $\operatorname{Re} T_{x}(g(x)) \geqslant$ $\alpha\|x\|, x \in B$, 则

$$
\frac{\left|T_{x}\left(D^{m} g(0)\left(x^{m}\right)\right)\right|}{m !} \leqslant 2(1-\alpha)\|x\|^{m}, \quad x \in B, \quad m=2,3, \cdots .
$$

上述估计式是精确的.

证 固定 $x \in B \backslash\{0\}$, 并记 $x_{0}=\frac{x}{\|x\|}$. 定义 $p(\xi)=\frac{1}{\xi} T_{x_{0}}\left(g\left(\xi x_{0}\right)\right), \xi \in D$. 根据条件, 有 $p(\xi) \in H(D), \operatorname{Re} p(\xi)=\operatorname{Re} \frac{1}{|\xi|} T_{\xi x_{0}}\left(g\left(\xi x_{0}\right)\right) \geqslant \alpha, \xi \in D$, 且 $p(0)=$ $\lim _{\xi \rightarrow 0} \frac{1}{\xi} T_{x_{0}}\left(g\left(\xi x_{0}\right)\right)=T_{x_{0}}\left(x_{0}\right)=1$. 又因

$$
p(\xi)=1+\frac{T_{x}\left(D^{2} g(0)\left(x_{0}^{2}\right)\right)}{2 !} \xi+\cdots+\frac{T_{x}\left(D^{m} g(0)\left(x_{0}^{m}\right)\right)}{m !} \xi^{m-1}+\cdots, \quad \xi \in D,
$$

由引理 1 , 可推出

$$
\frac{\left|T_{x}\left(D^{m} g(0)\left(x^{m}\right)\right)\right|}{m !} \leqslant 2(1-\alpha)\|x\|^{m}, \quad x \in B, \quad m=2,3, \cdots .
$$


证毕.

不难验证 $g(x)=\frac{1-(1-2 \alpha) T_{u}(x)}{1+T_{u}(x)} x(x \in B)$ 满足引理 5 的条件, 其中 $u \in \partial B$. 取 $x=r u(0 \leqslant r<1)$, 则 $(1.3)$ 式中等号成立.

由引理 3 , 也可证明

引理 6 设 $g \in H(B), g(0)=0, D g(0)=I$, 且 $\alpha \in(0,1)$. 若 $\left|\frac{T_{x}(g(x))}{\|x\|}-\frac{1}{2 \alpha}\right|<$ $\frac{1}{2 \alpha}, x \in B \backslash\{0\}$, 则

$$
\frac{\left|T_{x}\left(D^{m} g(0)\left(x^{m}\right)\right)\right|}{m !} \leqslant 2(1-\alpha)\|x\|^{m}, \quad x \in B, \quad m=2,3, \cdots .
$$

当 $m=2$ 时, 上述估计式是精确的.

证 固定 $x \in B \backslash\{0\}$, 并记 $x_{0}=\frac{x}{\|x\|}$. 定义 $p(\xi)=\frac{2 \alpha}{\xi} T_{x_{0}}\left(g\left(\xi x_{0}\right)\right)-1, \xi \in D$. 根据条件, 有 $p(\xi) \in H(D), p(0)=2 \alpha-1$, 且 $p(D) \subset D$. 又因

$p(\xi)=2 \alpha-1+\frac{2 \alpha T_{x}\left(D^{2} g(0)\left(x_{0}^{2}\right)\right)}{2 !} \xi+\cdots+\frac{2 \alpha T_{x}\left(D^{m} g(0)\left(x_{0}^{m}\right)\right)}{m !} \xi^{m-1}+\cdots, \quad \xi \in D$,

由引理 3 得

$$
\frac{2 \alpha\left|T_{x}\left(D^{m} g(0)\left(x_{0}^{m}\right)\right)\right|}{m !} \leqslant 1-(2 \alpha-1)^{2}
$$

即

$$
\frac{\left|T_{x}\left(D^{m} g(0)\left(x^{m}\right)\right)\right|}{m !} \leqslant 2(1-\alpha)\|x\|^{m}, \quad x \in B, \quad m=2,3, \cdots .
$$

证毕.

不难验证 $g(x)=\frac{\left(1-T_{u}(x)\right) x}{1+(1-2 \alpha) T_{u}(x)}(x \in B)$ 满足引理 6 的条件, 其中 $u \in \partial B$. 取 $x=r u(0 \leqslant r<1)$, 则当 $m=2$ 时, (1.4) 式中等号成立.

引理 $7^{[8]}$ 设 $f$ 是将 $B$ 映到另一个复 Banach 空间的正规化局部双全纯映 射, 且 $\varepsilon \in[0,1]$, 则 $f$ 是正规化双全纯 $\varepsilon$ 星形映射的充要条件是

$\operatorname{Re}\left\{T_{x}\left[(D f(x))^{-1}(f(x)-\varepsilon f(y))\right]\right\} \geqslant 0, \quad \forall x, y \in B, \quad\|y\| \leqslant\|x\|<1$.

引理 $\mathbf{8}^{[14]}$ 若 $\varphi(x) \in H(B, B)$, 则

$$
\frac{\left\|D^{m} \varphi(0)\left(x^{m}\right)\right\|}{m !} \leqslant\|x\|^{m}, \quad x \in B, \quad m=1,2, \cdots .
$$

引理 $\mathbf{9}^{[12]}$ 设 $p(z)$ 是一个至多 $n$ 次的多项式, 且 $|p(z)| \leqslant 1, z \in \bar{D}$, 则

$$
\left|p^{\prime}(z)\right| \leqslant n, \quad z \in \bar{D} \text {. }
$$

由引理 9 , 可证下面的

引理 10 设 $H_{n}(\xi, x)=p_{0}(x)+p_{1}(x) \xi+\cdots+p_{n}(x) \xi^{n}, \xi \in \mathbb{C}$, 其中 $p_{j}(x) \in$ $H(\bar{B}, X), j=0,1, \cdots, n$. 固定 $x \in \bar{B}$, 若 $\left\|H_{n}(\xi, x)\right\| \leqslant 1, \xi \in \bar{D}$, 则

$$
\left\|\frac{\partial H_{n}(\xi, x)}{\partial \xi}\right\| \leqslant n, \quad \xi \in \bar{D} \text {. }
$$

证 固定 $\xi \in \mathbb{C} \backslash\{0\}, x \in \bar{B}$, 并记 $\xi_{0}=\frac{\xi}{|\xi|}$. 取 $y=\frac{\partial H_{n}(\xi, x)}{\partial \xi}$, 定义 $l(\eta)=$ $T_{y}\left(H_{n}\left(\eta \xi_{0}, x\right)\right), \eta \in \mathbb{C}$. 由条件知 $l(\eta)$ 是一个至多 $n$ 次的多项式, 且 $|l(\eta)|=$ $\left|T_{y}\left(H_{n}\left(\eta \xi_{0}, x\right)\right)\right| \leqslant\left\|H_{n}\left(\eta \xi_{0}, x\right)\right\| \leqslant 1, \eta \in \bar{D}$. 根据引理 9, 有

$$
\left|l^{\prime}(|\xi|)\right|=\left|T_{y}\left(\left.\frac{\partial H_{n}\left(\eta \xi_{0}, x\right)}{\partial \eta}\right|_{\eta=|\xi|}\right)\right|=\left\|\frac{\partial H_{n}(\xi, x)}{\partial \xi}\right\| \leqslant n, \quad \xi \in \bar{D} .
$$


证毕.

\section{2 定理的证明}

定理 1 的证 记 $g(z)=(D f(z))^{-1} f(z), z \in D^{n}$. 因 $f(z)=D f(z) g(z)$, 于是

$$
\begin{aligned}
& z+\frac{D^{k+1} f(0)\left(z^{k+1}\right)}{(k+1) !}+\cdots+\frac{D^{m} f(0)\left(z^{m}\right)}{m !}+\cdots \\
= & \left(I+\frac{D^{k+1} f(0)\left(z^{k}, .\right)}{k !}+\cdots+\frac{D^{m} f(0)\left(z^{m-1}, .\right)}{(m-1) !}+\cdots\right) \\
& \cdot\left(D g(0) z+\frac{D^{2} g(0)\left(z^{2}\right)}{2 !}+\cdots+\frac{D^{k+1} g(0)\left(z^{k+1}\right)}{(k+1) !}+\cdots+\frac{D^{m} g(0)\left(z^{m}\right)}{m !}+\cdots\right) .
\end{aligned}
$$

比较上式两边的齐次展开式可得

$$
D g(0) z=z, \quad D^{j} g(0)\left(z^{j}\right)=0, \quad j=2, \cdots, k .
$$

由 (2.1) 式得

$$
\frac{D^{m} f(0)\left(z^{m}\right)}{m !}=\frac{D^{m} g(0)\left(z^{m}\right)}{m !}+\frac{D^{m} f(0)\left(z^{m}\right)}{(m-1) !}, \quad m=k+1, \cdots, 2 k,
$$

即

$$
\frac{D^{m} f(0)\left(z^{m}\right)}{m !}=\frac{-1}{m-1} \frac{D^{m} g(0)\left(z^{m}\right)}{m !}, \quad m=k+1, \cdots, 2 k .
$$

因 $f(z)$ 是 $D^{n}$ 上的 $\alpha$ 次殆星形映射, 故 $g(z)$ 满足引理 2 的条件. 根据引理 2 和 (2.2) 式得

$$
\begin{aligned}
\frac{\left\|D^{m} f(0)\left(z^{m}\right)\right\|}{m !} & =\frac{1}{m-1} \frac{\left\|D^{m} g(0)\left(z^{m}\right)\right\|}{m !} \\
& \leqslant \frac{2(1-\alpha)}{m-1}\|z\|^{m}, \quad z \in D^{n}, \quad m=k+1, \cdots, 2 k .
\end{aligned}
$$

证毕.

\section{不难验证}

$$
f(z)= \begin{cases}\left(z_{1} /\left(1-(1-2 \alpha) z_{1}^{k}\right)^{\frac{2(1-\alpha)}{(1-2 \alpha) k}}, z_{2}, \cdots, z_{n}\right)^{\prime}, & \alpha \in[0,1), \alpha \neq \frac{1}{2}, z \in D^{n}, \\ \left(z_{1} e^{z_{1}^{k} / k}, z_{2}, \cdots, z_{n}\right)^{\prime}, & \alpha=1 / 2, z \in D^{n}\end{cases}
$$

满足定理 1 的条件. 取 $z=(r, 0, \cdots, 0)^{\prime}(0 \leqslant r<1)$, 则当 $m=k+1$ 时, 定理 1 的 估计式是精确的.

注记 1 若 $f(z) \in S^{*}\left(D^{n}\right)$ (即 $\alpha=0$ ), 则有 $\|f(z)\| \geqslant\|z\| /(1+\|z\|)^{2}, z \in$ $D^{n}$ (参见文献 [15]). 由引理 2 得

注意到

$$
\|g(z)\|=\left\|(D f(z))^{-1} f(z)\right\| \leqslant \frac{\|z\|(1+\|z\|)}{1-\|z\|}, \quad z \in D^{n} .
$$

$$
\|f(z)\|=\|D f(z) g(z)\| \leqslant\|D f(z)\|\|g(z)\|,
$$

于是推出

$$
\|D f(z)\| \geqslant \frac{1-\|z\|}{(1+\|z\|)^{3}}, \quad z \in D^{n} .
$$


这表明 $D^{n}$ 上正规化双全纯星形映射偏差定理的下界估计式成立.

定理 2 的证 根据引理 4, 利用类似于定理 1 的证明方法, 可推出结论是正 确的. 证毕.

不难验证

$$
f(z)=\left(\frac{z_{1}}{\left(1-z_{1}^{k}\right)^{2(1-\alpha) / k}}, z_{2}, \cdots, z_{n}\right)^{\prime}, \quad z \in D^{n}
$$

满足定理 2 的条件. 取 $z=(r, 0, \cdots, 0)^{\prime}(0 \leqslant r<1)$, 则当 $m=k+1$ 时, 定理 2 的 估计式是精确的.

定理 3 的证 $\forall \lambda \in(0,1), \xi \in \bar{D} \backslash\{1\}$. 令 $g(x)=\lambda f(x)+(1-\lambda) f(\xi x), x \in B$. 因 $f(x) \in K(B)$, 于是 $g(B) \subset f(B)$. 定义 $\varphi(x)=f^{-1}(\lambda f(x)+(1-\lambda) f(\xi x)), x \in B$, 则 $\varphi \in H(B, B), \varphi(0)=0$, 且 $g(x)=f(\varphi(x))=\lambda f(x)+(1-\lambda) f(\xi x)$. 又因 $x=0$ 是 $f(x)-x$ 的 $k+1$ 阶零点, 故

$$
D \varphi(0) x=(\lambda+(1-\lambda) \xi) x, \quad D^{j} \varphi(0)\left(x^{j}\right)=0, \quad j=2, \cdots, k .
$$

由 (2.3) 式得

$$
\begin{aligned}
& (\lambda+(1-\lambda) \xi)^{m} D^{m} f(0)\left(x^{m}\right)+D^{m} \varphi(0)\left(x^{m}\right) \\
= & \left(\lambda+(1-\lambda) \xi^{m}\right) D^{m} f(0)\left(x^{m}\right), \quad m=k+1, \cdots, 2 k .
\end{aligned}
$$

根据 (2.4) 式得

$$
D^{m} \varphi(0)\left(x^{m}\right)=\left(\lambda+(1-\lambda) \xi^{m}-(\lambda+(1-\lambda) \xi)^{m}\right) D^{m} f(0)\left(x^{m}\right) .
$$

再根据 (2.3) 和 (2.5) 式, 有

$$
\begin{aligned}
\varphi(x)= & (\lambda+(1-\lambda) \xi) x+\frac{D^{k+1} \varphi(0)\left(x^{k+1}\right)}{(k+1) !}+\cdots \\
& +\frac{\left(\lambda+(1-\lambda) \xi^{m}-(\lambda+(1-\lambda) \xi)^{m}\right) D^{m} f(0)\left(x^{m}\right)}{m !}+\cdots .
\end{aligned}
$$

不失一般性, 可设 $f \in H(\bar{B})$, 否则, 考虑 $f(r x) / r(0<r<1)$, 再令 $r \rightarrow 1-$. 固定 $x \in B \backslash\{0\}$, 并记 $x_{0}=x /\|x\|$, 则

$$
\begin{aligned}
1 \geqslant & \frac{1}{2 \pi} \int_{0}^{2 \pi}\left\|\varphi\left(e^{i \theta} x_{0}\right)\right\|^{2} d \theta \\
= & \frac{1}{2 \pi} \int_{0}^{2 \pi}\left\langle\varphi\left(e^{i \theta} x_{0}\right), \varphi\left(e^{i \theta} x_{0}\right)\right\rangle d \theta \\
= & \frac{\left|\lambda+(1-\lambda) \xi^{m}-(\lambda+(1-\lambda) \xi)^{m}\right|^{2}}{(m !)^{2}} \\
& \cdot \frac{1}{2 \pi} \int_{0}^{2 \pi}\left\langle D^{m} f(0)\left(\left(e^{i \theta} x_{0}\right)^{m}\right), D^{m} f(0)\left(\left(e^{i \theta} x_{0}\right)^{m}\right)\right\rangle d \theta \\
& +|\lambda+(1-\lambda) \xi|^{2}+p(p \geqslant 0) \\
\geqslant & \frac{\left|\lambda+(1-\lambda) \xi^{m}-(\lambda+(1-\lambda) \xi)^{m}\right|^{2}}{(m !)^{2}}\left\|D^{m} f(0)\left(x_{0}^{m}\right)\right\|^{2}+|\lambda+(1-\lambda) \xi|^{2}
\end{aligned}
$$


这蕴涵

$$
\frac{\left\|D^{m} f(0)\left(x_{0}^{m}\right)\right\|}{m !} \leqslant \frac{\left(1-|\lambda+(1-\lambda) \xi|^{2}\right)^{1 / 2}}{\left|\lambda+(1-\lambda) \xi^{m}-(\lambda+(1-\lambda) \xi)^{m}\right|} .
$$

取 $\lambda=1 / 2$, 由 (2.6) 式得

$$
\frac{\left\|D^{m} f(0)\left(x_{0}^{m}\right)\right\|}{m !} \leqslant \frac{\left(1-\left|\frac{1+\xi}{2}\right|^{2}\right)^{1 / 2}}{\left|\frac{1+\xi^{m}}{2}-\left(\frac{1+\xi}{2}\right)^{m}\right|} .
$$

令 $\xi=e^{i \theta}(\theta \in \mathbb{R}, \theta \neq \pm 2 j \pi, j \in \mathbb{N})$, 于是

$$
\begin{aligned}
\frac{\left\|D^{m} f(0)\left(x_{0}^{m}\right)\right\|}{m !} & \leqslant \frac{\left|\sin \frac{\theta}{2}\right|}{\left|\frac{1+\cos m \theta-2\left(\cos \frac{\theta}{2}\right)^{m} \cos \frac{m \theta}{2}}{2}+\frac{\sin m \theta-2\left(\cos \frac{\theta}{2}\right)^{m} \sin \frac{m \theta}{2}}{2} i\right|} \\
& =\frac{\left|\sin \frac{\theta}{2}\right|}{\sqrt{\frac{1+\cos m \theta}{2}-2\left(\cos \frac{\theta}{2}\right)^{m} \cos \frac{m \theta}{2}+\left(\cos \frac{\theta}{2}\right)^{2 m}}} \\
& =\frac{\left|\sin \frac{\theta}{2}\right|}{\left|\cos \frac{m \theta}{2}-\left(\cos \frac{\theta}{2}\right)^{m}\right|},
\end{aligned}
$$

其中 $i=\sqrt{-1}$. 取 $\theta=2 \pi / m$, 则有

$$
\frac{\left\|D^{m} f(0)\left(x^{m}\right)\right\|}{m !} \leqslant \frac{\sin \frac{\pi}{m}}{1+\left(\cos \frac{\pi}{m}\right)^{m}}\|x\|^{m}, \quad x \in B, \quad m=k+1, \cdots, 2 k .
$$

证毕.

设 $f(x)=\frac{x}{1-\langle x, u\rangle}, x \in B$, 其中 $u \in \partial B$. 根据文献 [16] 知 $f(x) \in K(B)$. 取 $x=r u(0 \leqslant r<1), u \in \partial B$, 则当 $m=2$ (即 $k=1)$ 时, 可推出定理 3 的估计式是 精确的.

定理 4 的证 根据引理 5, 利用类似于定理 1 的证明方法，可推出结论是正 确的. 证毕.

设

$$
f(x)= \begin{cases}\frac{x}{\left(1-(1-2 \alpha)\left(T_{u}(x)\right)^{k}\right)^{\frac{2(1-\alpha)}{(1-2 \alpha) k}},} & \alpha \in[0,1), \alpha \neq 1 / 2, x \in B, \\ x e^{\left(T_{u}(x)\right)^{k} / k}, & \alpha=1 / 2, x \in B,\end{cases}
$$

其中 $u \in \partial B$. 显见 $f(x)$ 是 $B$ 上的正规化全纯映射，且 $x=0$ 是 $f(x)-x$ 的 $k+1(k \in \mathbb{N})$ 阶零点. 当 $\alpha \in[0,1), \alpha \neq 1 / 2$ 时，直接计算表明

$$
\begin{gathered}
D f(x)=\frac{\left(1-(1-2 \alpha)\left(T_{u}(x)\right)^{k}\right) I+2(1-\alpha)\left(T_{u}(x)\right)^{k-1} x T_{u}(.)}{\left(1-(1-2 \alpha)\left(T_{u}(x)\right)^{k}\right)^{\frac{2(1-\alpha)}{k(1-2 \alpha)}+1}}, \\
(D f(x))^{-1}=\left(1-(1-2 \alpha)\left(T_{u}(x)\right)^{k}\right)^{\frac{2(1-\alpha)}{k(1-2 \alpha)}}\left(I-\frac{2(1-\alpha)\left(T_{u}(x)\right)^{k-1} x T_{u}(.)}{1+\left(T_{u}(x)\right)^{k}}\right),
\end{gathered}
$$

其中 $x T_{u}(\cdot)$ 是将 $X$ 映到 $X$ 内的有界线性算子. 因此 $f(x)$ 是 $B$ 上的局部双全纯 映射.

$$
\operatorname{Re}\left(\frac{T_{x}\left((D f(x))^{-1} f(x)\right)}{\|x\|}-\alpha\right)=(1-\alpha) \operatorname{Re} \frac{1-\left(T_{u}(x)\right)^{k}}{1+\left(T_{u}(x)\right)^{k}}>0, \quad x \in B \backslash\{0\} .
$$


当 $\alpha=1 / 2$ 时, 同理可证上式成立. 由定义 1 得 $f(x)$ 是 $B$ 上的 $\alpha(0 \leqslant \alpha<1)$ 次 殆星形映射. 取 $x=r u(0 \leqslant r<1), u \in \partial B$, 则当 $m=k+1$ 时, 可推出定理 4 的 估计式是精确的.

定理 5 的证 根据引理 6 , 利用类似于定理 1 的证明方法, 可推出结论是正 确的. 证毕.

设

$$
f(x)=\frac{x}{\left(1-\left(T_{u}(x)\right)^{k}\right)^{2(1-\alpha) / k}}, \quad x \in B, \quad 0<\alpha<1,
$$

其中 $u \in \partial B$. 显见 $f(x)$ 是 $B$ 上的正规化全纯映射，且 $x=0$ 是 $f(x)-x$ 的 $k+1(k \in \mathbb{N})$ 阶零点. 直接计算表明

$$
\begin{gathered}
D f(x)=\frac{\left(1-\left(T_{u}(x)\right)^{k}\right) I+2(1-\alpha)\left(T_{u}(x)\right)^{k-1} x T_{u}(\cdot)}{\left(1-\left(T_{u}(x)\right)^{k}\right)^{\frac{2(1-\alpha)}{k}+1}} \\
(D f(x))^{-1}=\left(1-\left(T_{u}(x)\right)^{k}\right)^{\frac{2(1-\alpha)}{k}}\left(I-\frac{2(1-\alpha)\left(T_{u}(x)\right)^{k-1} x T_{u}(\cdot)}{1+(1-2 \alpha)\left(T_{u}(x)\right)^{k}}\right),
\end{gathered}
$$

其中 $x T_{u}(\cdot)$ 是将 $X$ 映到 $X$ 内的有界线性算子. 因此 $f(x)$ 是 $B$ 上的局部双全纯 映射. 注意到

$$
\operatorname{Re}\left(\frac{\|x\|}{T_{x}\left((D f(x))^{-1} f(x)\right)}-\alpha\right)=(1-\alpha) \operatorname{Re} \frac{1+\left(T_{u}(x)\right)^{k}}{1-\left(T_{u}(x)\right)^{k}}>0, \quad x \in B \backslash\{0\},
$$

即

$$
\left|\frac{1}{\|x\|} T_{x}\left((D f(x))^{-1} f(x)\right)-\frac{1}{2 \alpha}\right|<\frac{1}{2 \alpha}, \quad x \in B \backslash\{0\},
$$

由定义 2 知 $f(x) \in S_{\alpha}^{*}(B)(0<\alpha<1)$. 取 $x=r u(0 \leqslant r<1), u \in \partial B$, 则当 $m=k+1$ 时, 可推出定理 5 的估计式是精确的.

定理 6 的证 因 $f$ 是 $B$ 上的正规化双全纯 $\varepsilon$ 星形映射, 由引理 7 得

$$
\operatorname{Re}\left\{T_{x}\left[(D f(x))^{-1}(f(x)-\varepsilon f(y))\right]\right\} \geqslant 0, \quad\|y\| \leqslant\|x\|<1 .
$$

特别地, 有

$$
\operatorname{Re}\left\{T_{x}\left[(D f(x))^{-1}(f(x)-\varepsilon f(\xi x))\right]\right\} \geqslant 0, \quad x \in B, \quad \xi \in \bar{D} .
$$

固定 $\xi \in \bar{D}$, 定义 $g(x)=(D f(x))^{-1}(f(x)-\varepsilon f(\xi x)), x \in B$, 则 $g \in H(B), g(0)=0$, 且 $\operatorname{Re}\left[T_{x}(g(x))\right] \geqslant 0, x \in B$. 又因 $D f(x) g(x)=f(x)-\varepsilon f(\xi x)$, 于是

$$
\begin{aligned}
& (1-\varepsilon \xi) x+\frac{\left(1-\varepsilon \xi^{k+1}\right) D^{k+1} f(0)\left(x^{k+1}\right)}{(k+1) !}+\cdots+\frac{\left(1-\varepsilon \xi^{m}\right) D^{m} f(0)\left(x^{m}\right)}{m !}+\cdots \\
= & \left(I+\frac{D^{k+1} f(0)\left(x^{k}, \cdot\right)}{k !}+\cdots+\frac{D^{m} f(0)\left(x^{m-1}, \cdot\right)}{(m-1) !}+\cdots\right) \\
& \cdot\left(D g(0) x+\frac{D^{2} g(0)\left(x^{2}\right)}{2 !}+\cdots+\frac{D^{k} g(0)\left(x^{k}\right)}{k !}\right. \\
& \left.+\frac{D^{k+1} g(0)\left(x^{k+1}\right)}{(k+1) !}+\cdots+\frac{D^{m} g(0)\left(x^{m}\right)}{m !}+\cdots\right) .
\end{aligned}
$$


比较上述等式两边的齐次展开式, 有

$$
D g(0) x=(1-\varepsilon \xi) x, \quad D^{j} g(0)\left(x^{j}\right)=0, \quad j=2, \cdots, k .
$$

由 (2.7) 式得

$$
\frac{\left(1-\varepsilon \xi^{m}\right) D^{m} f(0)\left(x^{m}\right)}{m !}=\frac{(1-\varepsilon \xi) D^{m} f(0)\left(x^{m}\right)}{(m-1) !}+\frac{D^{m} g(0)\left(x^{m}\right)}{m !}, m=k+1, \cdots, 2 k,
$$

这蕴涵

$$
\begin{aligned}
& \frac{T_{x}\left(D^{m} f(0)\left(x^{m}\right)\right)}{m !} \\
= & -\frac{T_{x}\left(D^{m} g(0)\left(x^{m}\right)\right)}{\left(m-1-m \varepsilon \xi+\varepsilon \xi^{m}\right) m !}, \quad x \in B, \quad m=k+1, \cdots, 2 k .
\end{aligned}
$$

注意到 $D g(0) x=(1-\varepsilon \xi) x$, 取 $\xi=-1$, 则由引理 $5(\alpha=0$ 的情形) 和 (2.8) 式得

$$
\frac{\left|T_{x}\left(D^{m} f(0)\left(x^{m}\right)\right)\right|}{m !} \leqslant \frac{2(1+\varepsilon)\|x\|^{m}}{m-1+m \varepsilon+(-1)^{m} \varepsilon}, \quad x \in B, \quad m=k+1, \cdots, 2 k .
$$

证毕.

推论 1 的证 令 $\varepsilon=1$, 利用类似于定理 6 的证明方法, 可推出结论是正确 的. 证毕.

设

$$
f(x)=\frac{x}{1-T_{u}(x)}, \quad x \in B,
$$

其中 $u \in \partial B$. 显见 $f(x)$ 是 $B$ 上的正规化全纯映射. 直接计算表明

$$
\begin{gathered}
D f(x)=\frac{\left(1-T_{u}(x)\right) I+x T_{u}(\cdot)}{\left(1-T_{u}(x)\right)^{2}}, \\
(D f(x))^{-1}=\left(1-T_{u}(x)\right)\left(I-x T_{u}(\cdot)\right),
\end{gathered}
$$

其中 $x T_{u}(\cdot)$ 是将 $X$ 映到 $X$ 内的有界线性算子. 因此 $f(x)$ 是 $B$ 上的局部双全纯 映射. 注意到

$$
\operatorname{Re} T_{x}\left((D f(x))^{-1}(f(x)-f(\xi x))\right)=\operatorname{Re} \frac{(1-\xi)\left(1-T_{u}(x)\right)}{1-\xi T_{u}(x)}\|x\|, \quad \xi \in \bar{D},
$$

因 $g_{x}(\xi)=\frac{(1-\xi)\left(1-T_{u}(x)\right)}{1-\xi T_{u}(x)}\|x\| \in H(\bar{D})$, 故知 $\operatorname{Re} g_{x}(\xi)$ 在 $\bar{D}$ 上是调和的.

另一方面,

$$
\operatorname{Re} g_{x}\left(e^{i \theta}\right)=\frac{(1-\cos \theta)\left(1-\left|T_{u}(x)\right|^{2}\right)}{\left|1-e^{i \theta} T_{u}(x)\right|^{2}}\|x\| \geqslant 0, \quad x \in B
$$

根据调和函数的最小值原理, 有

$$
\operatorname{Re} T_{x}\left((D f(x))^{-1}(f(x)-f(\xi x))\right) \geqslant 0, \quad \xi \in \bar{D}, \quad x \in B .
$$

由定义 4 知 $f(x) \in Q(B)$. 取 $x=r u(0 \leqslant r<1), u \in \partial B$, 则当 $m=2$ (即 $k=1$ ) 时, 可推出推论 1 的估计式是精确的.

推论 2 的证 令 $\varepsilon=0$, 由定理 6 立即推出结论是正确的. 证毕.

关于推论 2 的估计式是精确的讨论同定理 4 . 
定理 7 的证 因 $h(x) \prec g(x)$, 故存在 $\varphi \in H(B, B), \varphi(0)=0$, 使得 $h(x)=$ $g(\varphi(x))$. 固定 $\xi \in \bar{D}$, 记 $\varepsilon_{k}=e^{2 k \pi i / m}, k=1,2, \cdots, m, m \in \mathbb{N}, H(\xi, x)=$ $f(\xi \varphi(x)), x \in B$, 其中 $i=\sqrt{-1}$. 因为

$$
W_{\xi}(x)=\frac{1}{m} \sum_{k=1}^{m} H\left(\xi, \varepsilon_{k} x\right)=\frac{1}{m} \sum_{k=1}^{m} f\left(\xi \varphi\left(\varepsilon_{k} x\right)\right) \in f(B),
$$

所以

$$
\begin{aligned}
W_{\xi}(x) & =\frac{1}{m} \sum_{k=1}^{m} \sum_{j=1}^{\infty} H_{j}\left(\xi, \varepsilon_{k} x\right) \\
& =\sum_{j=1}^{\infty}\left(\frac{1}{m} \sum_{k=1}^{m} H_{j}(\xi, x)\left(\varepsilon_{k}\right)^{j}\right) \\
& =\sum_{j=1}^{\infty}\left(H_{j}(\xi, x) \frac{1}{m} \sum_{k=1}^{m}\left(\varepsilon_{k}\right)^{j}\right) \\
& =H_{m}(\xi, x)+H_{2 m}(\xi, x)+\cdots,
\end{aligned}
$$

其中 $H_{m}(\xi, x)(m=1,2, \cdots)$ 是关于 $x$ 的 $m$ 次齐次多项式, 关于 $\xi$ 的至多 $m$ 次 多项式. 根据 (2.9) 式, 可定义 $\psi(x)=f^{-1}\left(W_{\xi}(x)\right), x \in B$. 因 $f \in K(B)$, 故 $\psi \in H(B, B), \psi(0)=0$, 且 $H_{m}(x, \xi)=D^{m} \psi(0)\left(x^{m}\right) / m !, m=1,2, \cdots$. 由引理 8 得

$$
\left\|H_{m}(\xi, x)\right\|=\frac{\left\|D^{m} \psi(0)\left(x^{m}\right)\right\|}{m !} \leqslant 1, \quad x \in \bar{B}, \quad m=1,2, \cdots .
$$

另一方面, 因 $g(x)=D f(x) x$, 于是 $g(\xi \varphi(x))=D f(\xi \varphi(x)) \xi \varphi(x)=\xi \sum_{m=1}^{\infty} \frac{\partial H_{m}(\xi, x)}{\partial \xi}$. 注意到

$$
\frac{D^{m} h(0)\left(x^{m}\right)}{m !}=\left.\frac{\partial H_{m}(\xi, x)}{\partial \xi}\right|_{\xi=1},
$$

根据引理 10 得

$$
\frac{\left\|D^{m} h(0)\left(x^{m}\right)\right\|}{m !} \leqslant m, \quad x \in \bar{B}, \quad m=1,2, \cdots,
$$

即

$$
\frac{\left\|D^{m} h(0)\left(x^{m}\right)\right\|}{m !} \leqslant m\|x\|^{m}, \quad x \in B, \quad m=1,2, \cdots .
$$

证毕.

设 $X$ 是具有内积 $\langle\cdot, \cdot\rangle$ 的复 Hilbert 空间. 由文献 [16] 知, 若 $u \in \partial B$, 则映射 $f(x)=\frac{x}{1-\langle x, u\rangle} \in K(B)$. 直接计算表明

取

$$
D f(x) x=\frac{x}{(1-\langle x, u\rangle)^{2}} .
$$

$$
\varphi(x)=x, \quad x=r u(0 \leqslant r<1), \quad u \in \partial B,
$$

则可推出定理 7 的估计式是精确的. 


\section{参考文献}

1 Gong S. The Bieberbach Conjecture. Providence: AMS-International Press, 1999. 126 176

2 Cartan H. Sur la possibilité d'étendre aux fonctions de plusieurs variables complexes la theorie des fonctions univalents. In: Montel P, ed. Lecons sur les Fonctions Univalents ou Mutivalents. Paris: GauthierVillar, 1933. 129 155

3 张文俊, 董道珍, 汪远征. Banach 空间中凸映照的增长定理. 数学季刊, 1991, 7(2): 84 87

4 Kohr G. On some best bounds for coefficients of several subclasses of biholomorphic mappings in $\mathbb{C}^{n}$. Complex Variables, 1998, 36: 261 284

5 Ropper K A, Suffridge T J. Convexity properties of holomorphic mappings in $\mathbb{C}^{n}$. Trans Amer Math Soc, 1999, 351: 1803 1833

6 Suffridge T J. Starlike and convex maps in Banach spaces. Pacific Jour of Math, 1973, 46: 575 589

7 Hamada H, Kohr G, Liczberski P. Starlike mappings of order $\alpha$ on the unit ball in complex Banach spaces. Glas Mat Ser III, 2001, 36: 39 48

8 Gong S, Liu T S. Criterion for the family of $\varepsilon$ starlike mappings. J Math Anal and Appl, 2002, 274: 696 704

9 刘太顺, 张文俊. 复 Banach 空间单位球上准凸映射的增长定理与掩盖定理. 中国科学, A 辑, 2002, 32(11): 1033 1041

10 林运泳, 洪毅. Banach 空间中全纯映射的若干性质. 数学学报, 1995, 38(2): 234 241

11 Boyd A V. Coefficient estimates for starlike functions of order $\alpha$. Proc Amer Math Soc, 1966, 17: $1016 \sim 1018$

12 Duren P L. Univalent Functions. New York: Springer-Verlag, 1983. 195 196

13 Graham I, Kohr G. Geometric Function Theory in One and Higher Dimensions. New York: Marcel Dekker, 2003. 34 35

14 Hamada H, Kohr G. Growth and distortion results for convex mappings in infinite dimensional spaces. Complex Variables, 2002, 47: 291 301

15 张文俊, 董道珍. Banach 空间中星形映照的增长定理与 $1 / 4$ 定理. 数学年刊, A 辑, 1992, 13(4): 417 423

16 Roper K A, Suffridge T J. Convex mappings on the unit ball of $\mathbb{C}^{n}$. J Anal Math, 1995, 65: 333 347 